\title{
Hall effects on field-aligned current generation in three-dimensional magnetic reconnection
}

\author{
N. Yokokawa ${ }^{1}$, M. Fujimoto ${ }^{1}$, Y. Yamade ${ }^{2}$, and T. Mukai ${ }^{3}$ \\ ${ }^{1}$ Department of Earth and Planetary Sciences, Tokyo Institute of Technology, Meguro, Tokyo 152-8551, Japan \\ ${ }^{2}$ Fuji Research Institute, Chiyoda, Tokyo 101-8443, Japan \\ ${ }^{3}$ Institute of Space and Astronautical Science, Sagamihara, Kanagawa 229-8510, Japan
}

(Received May 31, 2000; Revised January 29, 2001; Accepted February 28, 2001)

\begin{abstract}
Understanding the generation mechanisms of intense field-aligned currents (FACs) during auroral substorms is one of the central themes of magnetospheric physics. Three-dimensional (3D) magnetohydrodynamic (MHD) simulations of the Earth's magnetotail reconnection has been performed extensively to understand the global aspects of the generation mechanisms. In this study, however, by noting that the ion inertia length is not negligibly small compared to the current sheet thickness in the magnetotail, we investigate the FAC generation mechanism in the Hall-MHD system. With an idealized one-dimensional current sheet as the initial condition, we find that the FAC pattern obtained to be totally different from the MHD results. From a series of simulations, we also find that the predominance of this new FAC pattern is not necessarily due to the small thickness of the current sheet but is controlled more by the three-dimensionality of the system. The Hall-driven FAC dominates as long as the system is more two-dimensional-like than a critical value even for a relatively thick current sheet. Since this critical value turns out to be moderate, we conclude that reconnection in the Earth's magnetotail belongs to a general class of reconnection involving strong FACs generated by the Hall term.
\end{abstract}

\section{Introduction}

Upon a substorm onset, energy that has been stored in the lobe magnetic field is explosively converted into plasma kinetic energy, and magnetic reconnection is considered to be responsible for this fast energy conversion process in the Earth's magnetotail (e.g., Baker et al., 1996). The magnetotail reconnection produces earthward (tailward) jetting of hot plasma on the earthward (tailward) side of the reconnection site located presumably at 25-30 $R_{e}\left(R_{e}\right.$ : radius of the Earth) down the tail (Nagai et al., 1998). Bright aurorae that appear subsequent to an onset can be considered, from a global dynamics point of view, as results of generation of intense upward (from the ionosphere) field-aligned currents (FACs) carried by electrons accelerated downward into the ionosphere. Since reconnection can be considered as the engine for substorms, it is natural to question whether such intense upward FACs, so intense as to require significant downward field-aligned acceleration of electrons, can indeed be generated in the course of magnetotail reconnection. Knowledge on the importance of magnetic reconnection in the universe is increasing these days. With this in mind, the next question that comes up is how the mechanism operative in the magnetotail compares with those in other astrophysical situations.

As we are dealing with a large scale phenomena over the entire near-Earth tail, it may seem a natural choice to use the magnetohydrodynamics (MHD) framework for describ-

Copy right (c) The Society of Geomagnetism and Earth, Planetary and Space Sciences (SGEPSS); The Seismological Society of Japan; The Volcanological Society of Japan The Geodetic Society of Japan; The Japanese Society for Planetary Sciences. ing how FACs are generated in substorms. Indeed, threedimensional (3D) MHD simulations have been performed extensively to understand the global aspects of the dynamic phenomena (e.g., Scholer and Otto, 1991; Birn et al., 1996; Ugai and Wang, 1998). Once noting that the magnetotail current sheet is likely to become so thin prior to onsets that the ion inertia length cannot be taken as negligibly small, however, one begins to suspect whether the Hall term can really be neglected in describing the magnetotail reconnection.

In this paper, we will briefly review a study that focuses on the Earth's magnetotail parameters, in which the Hall effect is shown to modify the global FAC pattern significantly different from the MHD results. We proceed to a parameter survey and conclude that the predominance of the Hall-driven FAC as seen in the Earth's case does not necessarily require small thickness of the initial current sheet but is controlled more by the three-dimensionality of the system. For instance, even if the current sheet thickness $D$ is as large as a few tens times the ion inertia length, the scaling law obtained in this study suggests the Hall-FAC to dominate if the lateral extent of the reconnection line $R_{y}$ satisfies $R_{y} / D>7$. Since the required aspect ratio $R_{y} / D$ is not extremely large, we may conclude the universality of intense Hall-FAC generation in magnetic reconnection.

In this study, we will focus on an idealized geometry. Regarding the Earth's case, effects of the dipole field located at the earthward end of the current sheet will not be addressed. As such, the question is rather limited, however, the answer we reach is quite appealing in that a simple inclusion of the Hall term makes a new FAC system appear and even dom- 
inate as long as the system is moderately two-dimensional (2D)-like.

\section{Study on the Earth's Case}

In Hall-MHD, the well-known MHD equation for the electric field $\mathbf{E}=-\mathbf{V} \times \mathbf{B}$ is replaced by $\mathbf{E}=-\mathbf{V} \times \mathbf{B}+\mathbf{J} \times \mathbf{B} /($ en $)$ while others remain the same. Here the usage of the variables are as usual. In deriving the above equation, the electrons are assumed to be massless charge neutralizing fluid with zero temperature. Since the new equation can be rewritten as $\mathbf{E}=-\mathbf{V}_{e} \times \mathbf{B}$, where $\mathbf{V}_{e}$ is the electron bulk flow, now the magnetic field co-moves with the electrons while ions can make differential motions because of their finite inertia. The second (Hall) term becomes not negligible compared to the first (MHD) term when the velocity difference between ions and electrons is not negligibly small compared to the characteristic velocity scale. In other words, the Hall term becomes important when the spatial scale of interest is comparable to the relevant ion inertia length $\lambda_{i} \equiv V_{A} / \Omega_{i}$, where $V_{A}$ and $\Omega_{i}$ are the Alfven speed and the ion (proton) cyclotron frequency, respectively. Since $\lambda_{i} \sim 1,000 \mathrm{~km}$ in the Earth's case and the current sheet thickness $(D)$ just prior to an onset becomes much thinner than one Earth radius (e.g., Mitchell et al., 1990), it is not obvious at all that the Hall term can be neglected in the magnetotail situation.

Hereafter, we use the usual coordinates system in magnetospheric physics: The $x$ axis along the tail axis directed positive (negative) earthward (tailward), the $y$ axis directed positive from dawn to dusk, and the $z$ axis positive northward. Normalizations are made as follows: magnetic field by the lobe magnetic field $B_{0}(20 \mathrm{nT})$, density by the lobe density $n_{0}\left(0.05 \mathrm{~cm}^{-3}\right)$, velocity by the lobe Alfven velocity $V_{A}(2,000 \mathrm{~km} / \mathrm{s})$, time by the inverse of the proton cyclotron frequency in the lobe $\Omega_{i}{ }^{-1}(0.5 \mathrm{~s})$, and spatial scale by the lobe ion inertia length $\lambda_{i}(1,000 \mathrm{~km})$, respectively (Typical values are given in the parentheses). To study the magnetotail situation in this section, the initial current sheet thickness is set $D=1.5$.

The simulation box is $0<x<80 D,|y|<5 R_{y}$, and $0<$ $z<20 D$ ( $R_{y}$ is the lateral extent of the reconnection region as defined below). The initial magnetic field is modeled by

$$
\mathbf{B}(z)=\tanh (z / D) \mathbf{e}_{x}
$$

with the density profile

$$
n(z)=1+2 \operatorname{sech}^{2}(z / D)
$$

for $0<z<10 D$. This cross-tail current sheet located at $z=0$ with $j_{y}>0$ is sustained by ions flowing duskward $(+y$ direction). Another current sheet of reversed polarity

$$
\begin{aligned}
& \mathbf{B}(z)=-\tanh ((z-20 D) / D) \mathbf{e}_{x} \\
& n(z)=1+2 \operatorname{sech}^{2}((z-20 D) / D)
\end{aligned}
$$

for $10 D<z<20 D$ is situated for the sake of simple boundary conditions (see below). The ion temperature is initially uniform and the electron temperature is fixed to 0 . The ion beta in the lobe is 0.5 . Reconnection is initiated by localized anomalous resistivity in the $z=0$ current sheet.

$$
\begin{aligned}
& \eta(x, y, z)=\left(D / R_{m}\right)[4 S(x, y, z)+1] \\
& S(x, y, z)=\exp \left\{-\left(x / R_{x}\right)^{2}-\left(y / R_{y}\right)^{2}-\left(z / R_{z}\right)^{2}\right\}
\end{aligned}
$$

This ad-hoc assumption does not allow us to address the question of the reconnection mechanism. $R_{m}$ stands for the background magnetic Reynolds number and is taken to be 200. Note that $D$ is used as the characteristic scale in evaluating the above number. Symmetric boundary conditions are imposed at the $x$ and $z$ boundaries, while periodic boundary condition is taken in the $y$ direction. To be specific, symmetric boundary conditions for, say, $x=0$ are, $\partial n / \partial x=\partial P / \partial x=\partial B_{x} / \partial x=\partial V_{y} / \partial x=\partial V_{z} / \partial x=0$, and, $V_{x}=B_{y}=B_{z}=0$ and anti-symmetric across the $x=0$ plane. With the large enough simulation box, the boundary conditions have little effects except that the symmetry at $z=0$ suppresses the cross-tail K-H instability (the usage of massless electron model prohibits the kinetic kink instability as well, while this mode may play little role at the real mass ratio anyway) (e.g., Pritchett and Coroniti, 1996; Yoon et al., 1996; Daughton, 1998). $R_{x}=4 D, R_{y}=10(\sim 6.7 D)$, and $R_{z}=2 D$ are chosen. The grid sizes are $d x=0.6(0.4 D)$, $d y=1.0\left(0.1 R_{y}\right)$, and $d z=0.3(0.2 D)$, respectively. The simulation code utilizes 4 th order finite difference in calculating spatial derivatives and 4th order Runge-Kutta scheme for time integration. Viscosity corresponding to a Reynolds number of 200 (with $D$ as the characteristic scale length) is added to the equations of motion for ion fluid. Heating associated with this viscous as well as Joule dissipation is taken into account in the equation for ion pressure.

The effect of the Hall term to produce an additional current system in the proximity of the reconnection site has been known for a long time from a theoretical point of view (e.g., Sonnerup, 1979; Terasawa, 1983; Mandt et al., 1994; Hesse and Winske, 1994; Nakamura et al., 1998; Lottermoser et al., 1998; Nakamura and Fujimoto, 1998), and has been recently reported to be observed in the magnetotail (Oieroset et al., in preparation; Nagai et al., 1998). In order to see if this effect will modify the global FAC pattern, we have run a series of 2D Hall-MHD simulations to find that this additional current system can indeed be significant globally (Yamade $e t$ al., 2000). 2D simulations are started from the same initial condition as described above except that $R_{y}=\infty$. There is no FAC generation in 2D MHD. In Hall-MHD, the enhanced cross-tail current at the $\mathrm{X}$-line $(x, z)=(0,0)$ is carried by dawnward $(-y$ directed) flowing electrons. Since the field lines co-move with the electrons, field lines in the proximity of the reconnection region drift dawnward. These result in the generation of non-zero $B_{y}$ near the separatrices (positive at $z>0$, negative at $z<0$ ) (Mandt et al., 1994). As the field lines are convected earthward with the jet, field lines are piled-up and the cross-tail current is disrupted. The electrons come to co-move with the duskward ions, which bends the field lines in the opposite direction to result in the $B_{y}$ pattern of the reversed sense. A pair of FAC components of significant intensities is associated with these perturbations in the $B_{y}$ component and propagates from the reconnection region along the field lines in the $x$ direction. Measured on the cross-section plane located at as far as $10 R_{e}$ earthward of the X-line, these downward/upward (seen from the ionosphere, $+x /-x$ directed in the present coordinates) FAC densities peak at $>0.5 J_{0}$ for the range of the current sheet thickness relevant to the magnetotail $\left(D / \lambda_{i}=1-4\right)$, where $J_{0}$ is the initial cross-tail current density. This shows that 
the Hall current may contribute significantly to the global current pattern.

Now we proceed to 3D simulation results. Figure 1 shows ion flow (black arrows) as well as electron flow (red) patterns superposed on the color contour for the $B_{z}$ component at $z=0$. Flow arrows are shown only when their magnitudes exceed 0.25 . Ions approach the reconnection region from dawnside of it as they drift duskward $(+y)$ along $x=0$ and are accelerated earthward $(+x)$ by $(\mathbf{J} \times \mathbf{B})_{x}$. This, at the same time, means that the duskward flowing pressure carrying ions are disrupted at the dawnward edge of the reconnection region. Strong $B_{z}$ seen at the leading part of the jet indicates piling-up the field lines there.

Electrons are showing enhanced dawnward $(-y)$ acceleration on the duskside $(y>0)$ the reconnection region. As stated above, since the duskward flow of ions are disrupted on the dawn-edge of the reconnection region and pressure carrying ions are depleted in this duskside, the current sheet is thinned and the current density is increased. It has to be the dawnward electrons that sustain this intensified crosstail current. At the leading part of the jet where the fieldlines are piling-up, on the other hand, electrons are deflected duskward $(+y)$. With the cross-tail current being disrupted in this part, electrons are simply co-moving with duskward ions. As such, the electron flow pattern at $0<y<R_{y}$ is essentially the same as that seen in $2 \mathrm{D}$, that is, dawnward near the reconnection region and then duskward as the field lines pile-up in the leading part of the jet. Enhanced current sheet thinning and enhanced convection of the lobe field lines towards $z=0$ takes place on the duskside of the reconnection region. Adding this effect with the dawnward electron flow in the same region results in preferential transport of dusklobe field lines into the reconnection region (Nakamura and

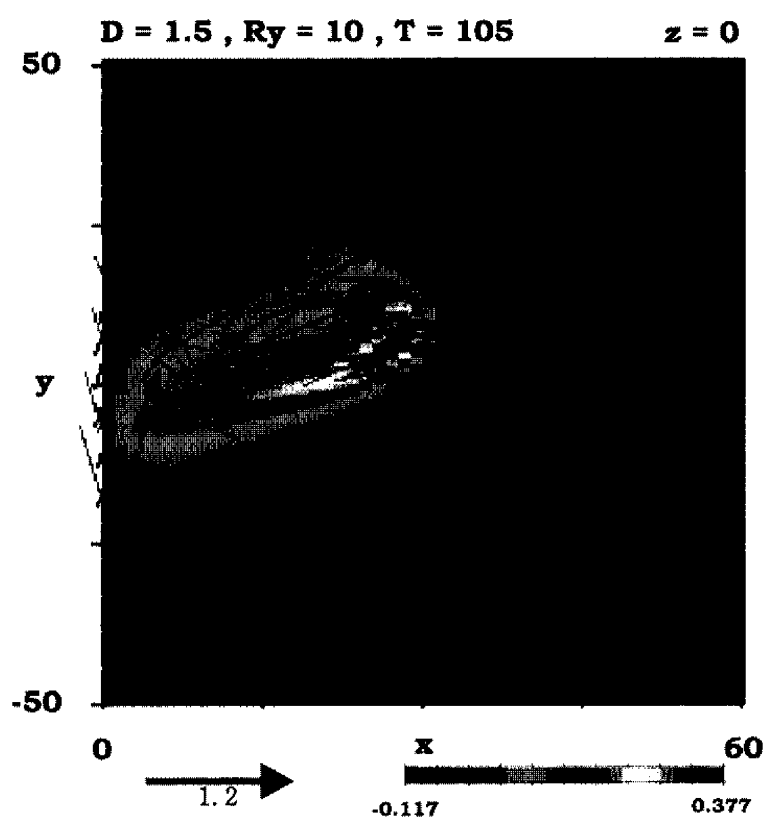

Fig. 1. $B_{z}$ component (color contour), the ion flow (black arrows), and the electron flow (red arrows) on the $z=0$ plane. Dawnward-then-duskward deflection of electrons at $0<y<R_{y}(=10)$ is quite similar to that seen in 2D Hall-MHD.
Fujimoto, 1998).

As stated above, the majority of the reconnected field lines are coming from the duskside lobe and are subject to dawnward-then-duskward $(-y$ then $+y$ directed) deflection due to the Hall effect. (It would be worth noting that such a deflection of the jet is not seen in MHD results. See the next section.) Now we proceed to inspect the resultant FAC pattern. Two panels in Fig. 2 show the cross-section $(y-z)$ plane at $x=30$ and the meridional $(x-z)$ cut at $y=0$ of FAC pattern at $T=105$, when the reconnection has fully developed. As expected from the fact that the electron flow pattern around the tail axis $(y=0)$ on the $z=0$ plane showed essentially the same feature as in $2 \mathrm{D}$, the field lines in the proximity of the $y=0$ meridional plane are bent similarly to the 2D situation and the resultant FAC pattern shown in Fig. 2(b) is essentially the same as that obtained in the 2D simulation. There are downward FAC (into the ionosphere away from the $\mathrm{X}$-line, directed $+x$ in the present coordinates: red) located at the highest-latitude (largest $|z|$ ) edge of the current sheet and upward FAC (from the ionosphere, directed $-x$ : blue) at lower-latitude adjacent to it. The cross-section slice in Fig. 2(a) shows that the FAC pattern on this plane is totally different from that in MHD (Yamade et al., 2000). A pair of upward and downward current patches is known to appear in MHD. This FAC pair is anti-symmetric about the $y=0$ meridional plane, with upward on duskside $(y>0)$ and downward on dawnside $(y<0)$ (e.g., Scholer and Otto, 1991; Birn et al., 1996; Ugai and Wang, 1998). In contrast, FACs in Fig. 2(a) are layered in latitude (along the $z$ axis) around the midnight meridian $y=0$.
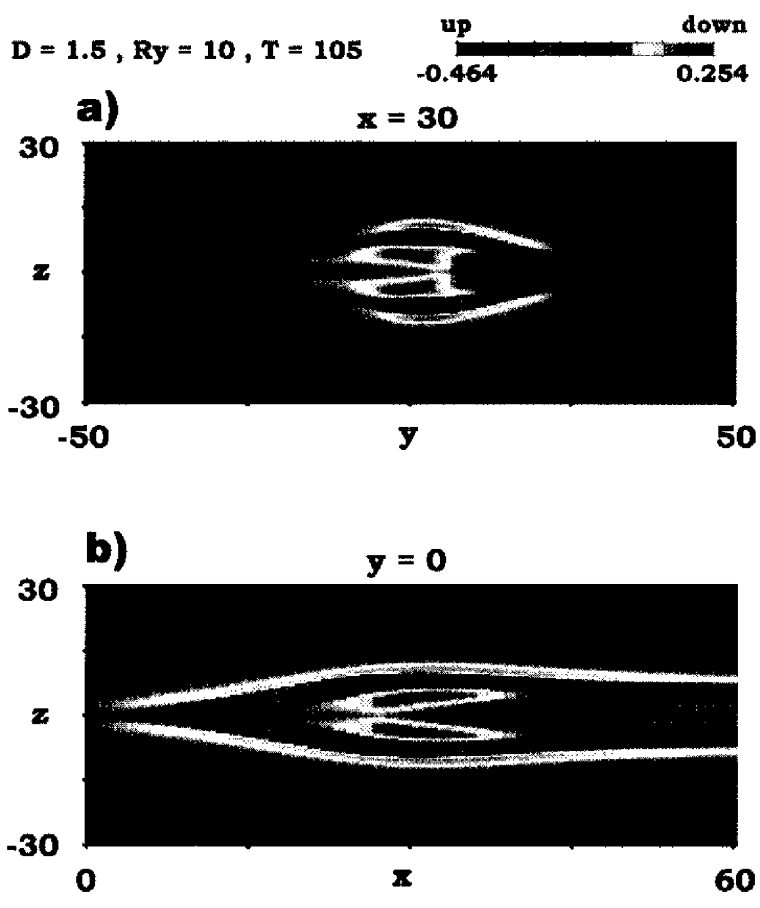

Fig. 2. FAC density pattern taken on (a) the cross-section plane at $x=30$ and (b) the meridional plane at $y=0$. Negative (blue) indicates upward FAC from the ionosphere (directed $-x$ ), and vice versa. Panel (b) shows structure similar to the $2 \mathrm{D}$ case, that is, downward (red) at the highest-latitude and upward (blue) located adjacent to it. FAC pattern on the cross-section slice in Panel (a) is totally different from MHD results. 
Substituting parameters for the Earth's magnetotail, the location of this slice is $5 R_{e}$ earthward of the X-line and thus shows that the modification by the Hall term is significant over a global scale. The peak upward current density is 0.46 in $e n_{0} V_{A}$ unit $\left(0.7 J_{0}\right)$ and the total upward current flowing across this plane is $10 e n_{0} V_{A} \lambda_{i}{ }^{2}$. These are translated to $7 n \mathrm{~A} / \mathrm{m}^{2}$ and $1.5 \times 10^{5} \mathrm{~A}$, respectively, for the Earth's magnetotail and are of significant amounts in the context of magnetospheric physics. The peak upward FAC density in Fig. 2(a) is the maximum value at this time over the simulation box. As reconnection develops, the maximum is seen to propagate earthward $(+x)$ from the X-line and reaches the $x=30$ plane at the sampling time of Fig. $2, T=105$. The FAC pattern on the cross-section plane including the maximum stays essentially the same as it propagates further earthward. When it eventually hits the ionosphere (not included in the present study), it is expected to give rise to a new additional path of magnetosphere-ionosphere coupling via Hall-FAC, which seems to be containing significant amount of current but is out of the scope of previous global MHD simulations.

\section{Parameter Survey}

In the previous section, it is shown that the Hall term has significant effects on the FAC generation in the Earth's magnetotail reconnection. The key argument at the starting point of the previous section is the small thickness of the magnetotail current sheet, as thin as comparable to the relevant ion inertia length $(D=1.5)$. In this section, we will show that the small thickness is not necessary but the predominance of the Hall-FAC is controlled more by the aspect ratio $R_{y} / D$ of the reconnection region, in other words, the threedimensionality of the system.

In order to study the above point, we have performed a series of simulations by varying the parameters $D$ and $R_{y}$. Other parameters are varied accordingly. To be specific, the size of the diffusion region $\left(R_{x}, R_{z}\right)=(4 D, 2 D)$, the size of the simulation box $\left(L_{x}, L_{y}, L_{z}\right)=\left(80 D, 10 R_{y}, 20 D\right)$ or larger if necessary, and the grid sizes $(d x, d y, d z)=$ $\left(0.4 D, 0.1 R_{y}, 0.2 D\right)$ or smaller if necessary, respectively.

First we vary the current sheet thickness $D$ while keeping the lateral extent of the reconnection region $R_{y}=10$. Figure 3 compares the FAC pattern on the $x=20 D$ cross-tail section at $T=70 D$. The panel at the top is the same as shown in Fig. 2(a). As $D$ is increased, one can see that another patch of upward FAC (blue) start to appear on the duskside at lower latitude $(y>0$, smaller $|z|)$. For $D>3$, the intensity within the duskside patch becomes comparable to the high-latitude Hall-FAC. When paired with the dawnside downward FAC patch (red, $y<0$ ), the lower latitude patches look similar to the MHD pattern. Indeed, while the electron flow pattern at the leading part of the reconnection jet is mostly unidirectionally duskward (directed $+y$ ) at $D=1.5$, the dawnside part of the jet becomes to have dawnward $(-y)$ flow components as $D$ is increased and the jet is clearly bifurcated in the dawn-dusk direction at $D>3$. Figure 4 is the same as Fig. 1 except that it is now for results with $D=6$. The bifurcated electron flow creating a bow-like pattern in the $B_{z}$ color contour carries magnetic flux to dawnside. This bifurcated flow, which is indeed seen in MHD simulations, bends the field lines to cause the MHD-like FAC pattern (e.g., Birn

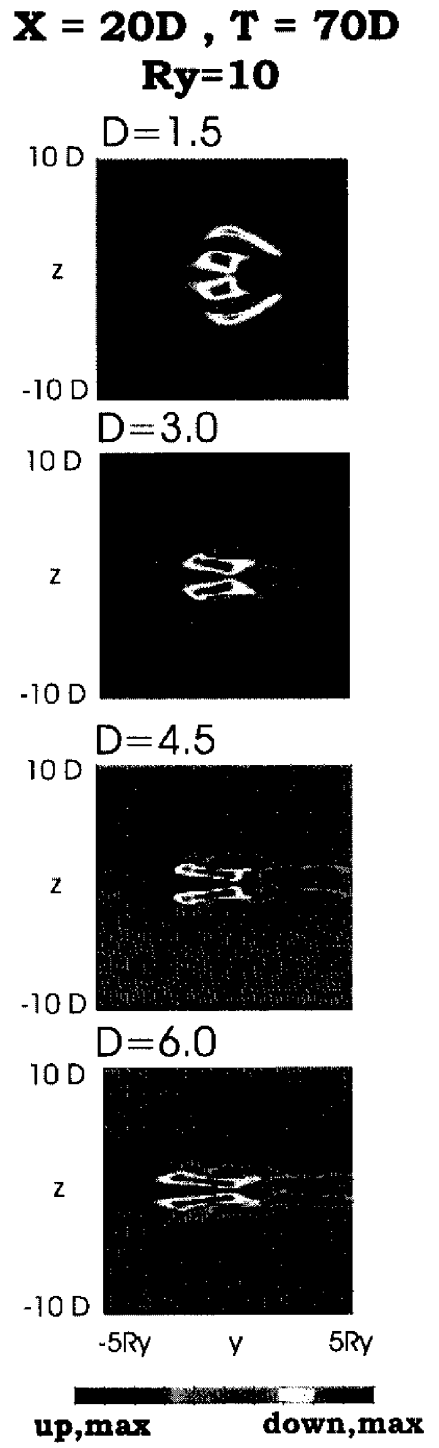

Fig. 3. Comparison of FAC patterns on the cross-section slice for various current sheet thickness $D$ with fixed lateral extent of the reconnection region $R_{y}=10$. At $D>3$, structure at lower-latitude (smaller $|z|$ ) is quite similar to that obtained in MHD.

and Hesse, 1996).

This additional bending of the field lines is best depicted in the $B_{y}$ pattern on the cross-tail section shown in Fig. 5. Note that the background magnetic field in the northern hemisphere $(z>0)$ is directed earthward $\left(B_{x}>0\right)$ so that dawnward (duskward) displacement of the $z=0$ crossing point of the field line results in positive (negative) $B_{y}$, and that the FACs discussed here can be roughly estimated by $J_{x} \sim-\partial B_{y} / \partial z$ (positive: downward, negative: upward). For $D=1.5$, two major patches appear in the northern hemisphere $(z>0)$, positive on the duskside $(y>0)$, negative at midnight $(y \sim 0)$, and these result from dawnward-thenduskward deflection of the electron flow within the reconnection jet. The upward FACs are located between the two patches while two downward FAC regions are located at the upper edge of the positive $B_{y}$ and the lower edge of the negative $B_{y}$. For $D=6$, in addition to the above structure, two new patches appear at lower-latitude (smaller $z$ ), pos- 


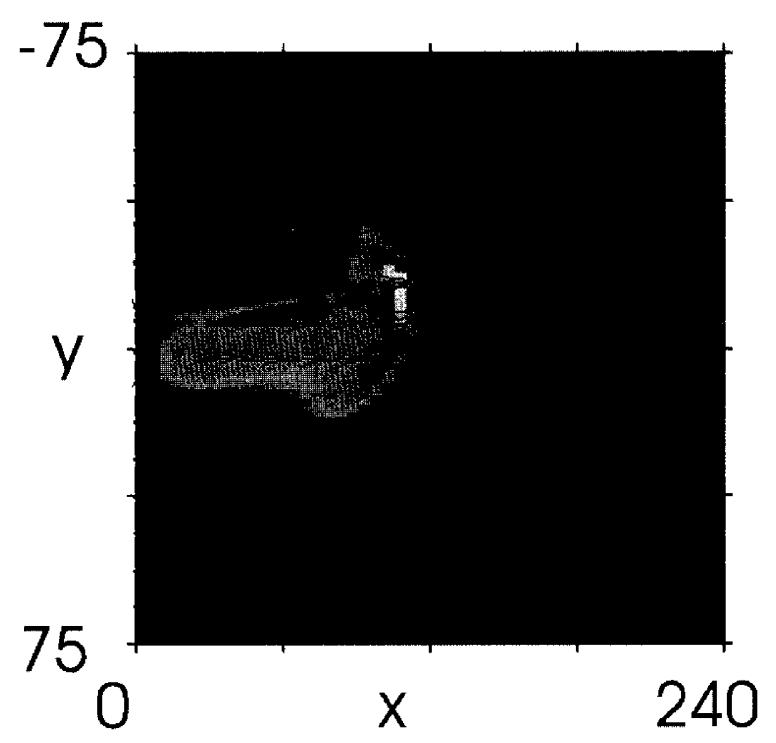

Fig. 4. The same as Fig. 1 but for the $D=6$ and $R_{y}=10$ case. Bow-like shape of the $B_{z}$ color contour is a result of electron flow bifurcation and indicates how MHD-like FAC pattern is generated for this case.

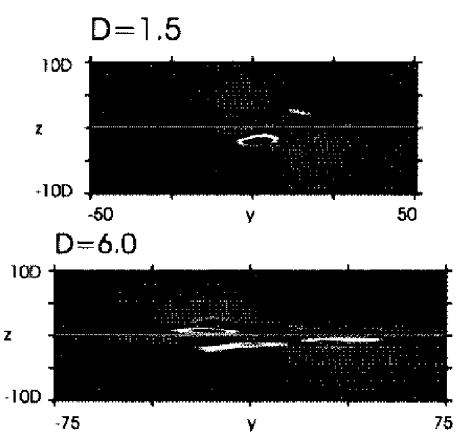

Fig. 5. $\quad B_{y}$ component on the same cross-section slice for $D=1.5$ and 6 . Pattern seen for $D=1.5$ is due to field-line bending associated with the electron flow deflection seen in Fig. 1. The newly emerging pattern at lower-latitude for $D=6$ is due to dawn-dusk bifurcation of the electron flow at the leading part of the jet, giving rise to MHD-like FAC pattern in Fig. 3.

itive on the dawnside and negative on the duskside. This newly appearing pattern is due to the field line bending associated with the jet-bifurcation. Gradient at the upper edge of each patch is the source for FAC that shows the MHD-like pattern. One can see dawn-dusk elongation of the dawnside downward FAC patch ( $y<0$, red) as $D$ is increased (Fig. 3). This deformation reflects the shift in the dominant generation mechanism.

Enhanced jet bifurcation with increasing $D$ generates stronger MHD-like current patterns, however, one should note that the high-latitude Hall-FAC is still comparable to this bifurcation generated component even with $D$ as large as 6 . This is because the thin current sheet in the proximity of the reconnection region is sustained by the dawnward $(-y)$ electron flow, which is still strong enough to produce electron flow deflection within the jet that generates intense high-latitude Hall-FAC.

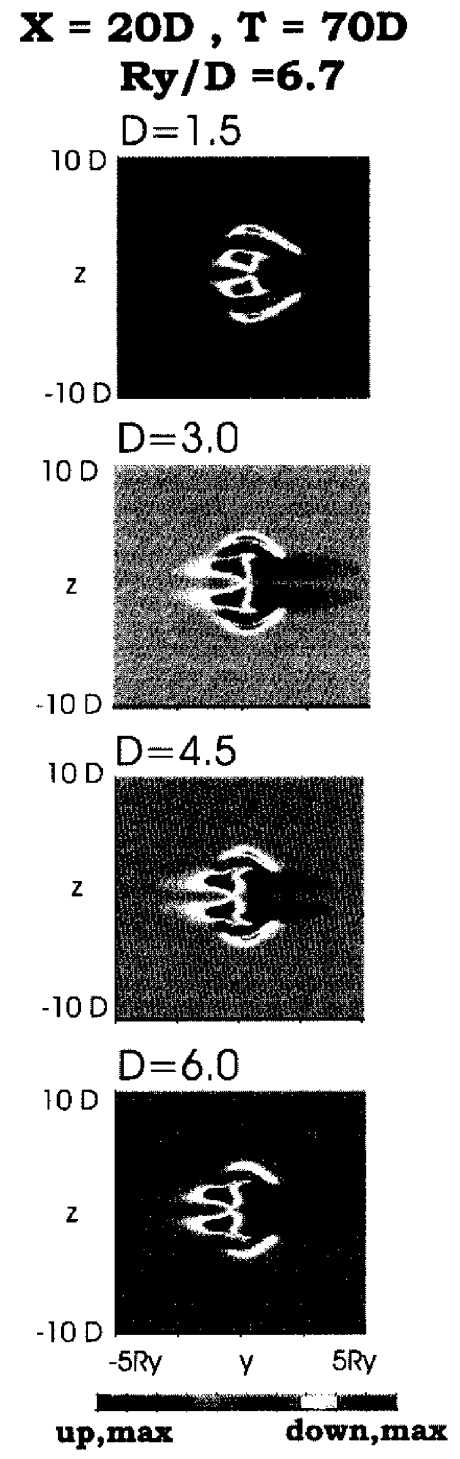

Fig. 6. Comparison of FAC patterns on the cross-section slice for various current sheet thickness $D$ with the fixed aspect ratio $R_{y} / D=6.7$. While $D$ is varied in the same range as Fig. 3, changes in the pattern are much reduced, indicating that the aspect ratio is an important parameter in determining the predominance of Hall-FAC.

From the above results, one might think that the MHDlike results are recovered as the current sheet thickness $D$ is increased and that $D$ is the controlling parameter of the significance of the Hall-FAC. On the other hand, by noting the appearance of the MHD-like FAC is due to the bifurcation at the front of the jet, one would think FAC pattern to depend strongly on the aspect ratio $R_{y} / D$ (three-dimensionality) of the system. (Note that FAC disappears in 2D MHD.) It is noted that increasing $D$ with $R_{y}$ fixed is not only changing the thickness but is also changing the aspect ratio to enhance the three-dimensionality of the system. Furthermore, in contrast to the FAC generation by the jet-bifurcation, it should be recalled that the Hall-FAC appears in 2D simulations as well, or rather, is strongest in the $2 \mathrm{D}$ situation where the dawndusk electron flow deflection is allowed to develop most. As such, the aspect ratio should be playing an important role in determining the significance of the Hall-FAC as well. 
In order to see how these two factors work to determine the FAC pattern, now we fix the aspect ratio $R_{y} / D=6.7$ and increase the thickness $D$. Results are shown in Fig. 6. While the current sheet thickness $D$ is varied in the same range as in Fig. 3, changes in the FAC pattern on the crosstail section is much reduced in this case. By inspecting the electron flow pattern on the $z=0$ plane, we find that the degree of bifurcation (as measured by the peak dawnward electron flow at the leading part of the jet) is enhanced with increasing thickness even if the aspect ratio is fixed. At this aspect ratio $R_{y} / D=6.7$, however, the effects of the electron flow deflection within the jet is more effective than the bifurcation at the leading part to generate stronger FAC at least up to $D=6$, leading us to conclude at this point that the aspect ratio of 6.7 is large enough (two-dimensional enough) for the high-latitude Hall-FAC to dominate at least up to $D=6$.

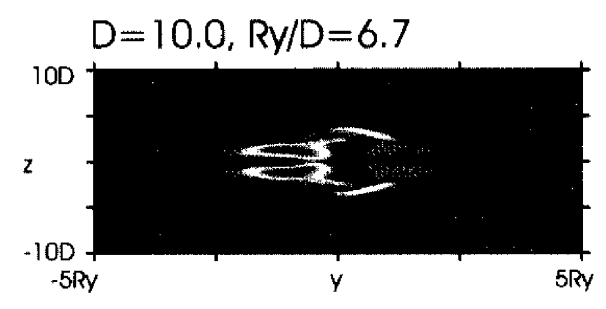

Fig. 7. FAC pattern on the cross-section slice for $D=10$ and $R_{y} / D=6.7$. Upward current intensity in the duskside MHD-like patch is becoming comparable to that from the high-latitude Hall-FAC at this $D$.

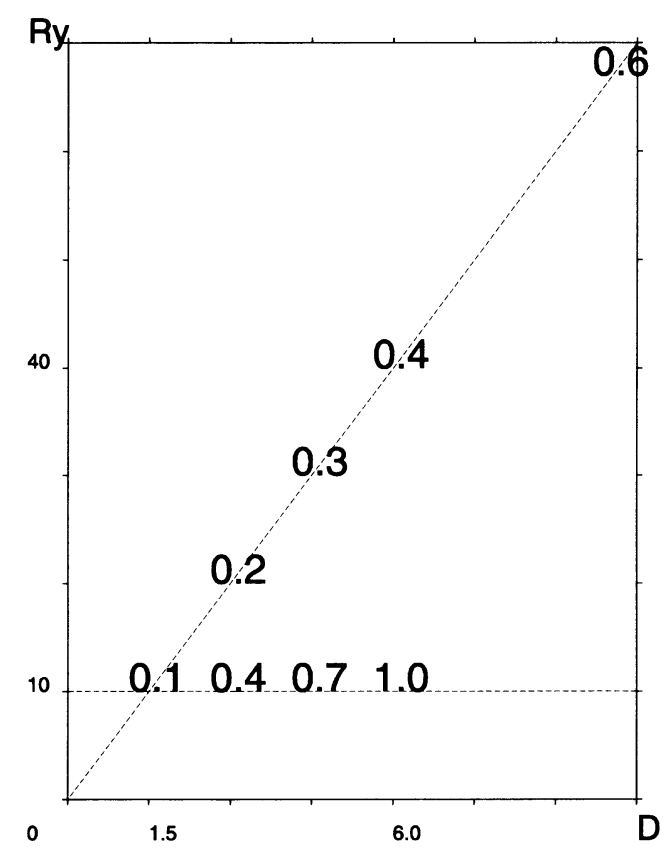

Fig. 8. A diagram showing the changes in the FAC pattern in a quantitative manner. The ratio between the peak intensity in the duskside MHD-like patch and that in the high-latitude Hall-FAC patch is shown. Results for the magnetic Reynolds number of $R_{m}=200$. The predominance of the Hall-MHD is enhanced with higher $R_{m}$, leading to a suggestion that Hall-FAC will dominate (at least) up to $D \sim 30$ as long as $R_{y} / D>7$ in space and astrophysical plasmas.
Figure 7 shows the FAC pattern for $D=10$ and $R_{y} / D=$ 6.7. At this $D$, one can see that the intensity within the patch of bifurcation generated upward current (located at smaller $|z|, y>0)$ is becoming comparable to the upward Hall-FAC. To show these gradual quantitative changes, the ratio between the peak intensity within the bifurcation-generated upward patch and that within the upward Hall-FAC patch is plotted in Fig. 8. When $R_{y}=10$ is fixed, the ratio becomes 1 at $D=6$. On the other hand, when the aspect ratio $R_{y} / D=6.7$ is fixed, the ratio increases with $D$ but is still less than unity $(0.6)$ at $D=10$. We also find that for $R_{m}=100 \sim 400\left(R_{m}\right.$ : background magnetic Reynolds number), the Hall-FAC peak intensity is proportional $R_{m}{ }^{1 / 2}$ while the bifurcation-generated FAC is rather insensitive to $R_{m}$ presumably because of its larger characteristic spatial scale. Using this peak current density ratio as a proxy for measuring the relative contribution of the Hall effects and MHD-like dynamics, for space plasmas with much larger $R_{m}$, we may come to a conclusion that the Hall-FAC is generally significant in magnetic reconnection: It remains significant (at least non-negligible) for a current sheet whose initial thickness is as large as a few tens times the relevant ion inertia length as long as the aspect ratio satisfies the moderate requirement of $>7$.

\section{Discussion}

The present results with the magnetotail-like parameters ( $D=1.5$ and $R_{y}=10$ ) suggest that the Hall current generated in the course of reconnection in the magnetotail is not only non-negligible but may contribute significantly to the global FAC pattern. MHD results for the Earth's case have been widely accepted because they have successfully reproduced the wedge-current pattern (e.g., McPherron et al., 1973), which has been inferred from and supported by vast amount of observations. Hall-MHD results for the Earth's case, on the other hand, fails to reproduce this feature. It should be noted here that the present simulations are done in an idealized geometry. Disappearance of the wedge-current pattern in the present results only means that it cannot be generated by reconnection in a one-dimensional current sheet. In MHD, wedge-current pattern is generated as the jet is bifurcated at its leading part. The bifurcation is likely to occur also in Hall-MHD as the jet collides with the dipole field located at the earthward end of the current sheet. Birn and Hesse (1996) have treated this process in MHD but it would be worth re-inspecting the process by Hall-MHD. The reinspection is crucial for understanding the FAC generation in magnetotail reconnection involving a thin current sheet. On the other hand, previous results (e.g., Scholer and Otto, 1991; Ugai and Wang, 1998; Yamade et al., 2000) show that the present boundary condition well simulates the obstacle effect of the dipole field for MHD models, and be this the case for the Hall-MHD as well, there would arise a serious question concerning the origin of the wedge-current pattern.

Collision of the jet with the dipole will not smear out the high-latitude pair of upward/downward currents that is driven by the Hall effects, since they are generated by an independent mechanism (electron flow deflection within the jet) in the current sheet remote from the dipolar region. Estimates in the previous section show that the upward current is indeed strong, leading to the prediction of a new FAC system 
located at high-latitude in the midnight sector that is out of the scope of MHD treatments. It is noted that the downward Hall-FACs at the highest latitude have been identified recently from spacecraft observations (Fujimoto et al., 1997, 2001).

Since the dawn-dusk extent of the reconnection region is an unknown quantity, one has the freedom to think of a smaller $R_{y}$ for the Earth's case, which may increases the three-dimensionality of the system and work to recover the wedge-current pattern even in the absence of the dipole field. It turns out, however, that there is not much room for $R_{y}$ to become smaller if one wants to have reconnection to so fully develop as to be associated with an Alfvenic jet. We find the jet velocity to be reduced for $R_{y}<7$. This can be understood by recalling the fact that ions are accelerated by $(\mathbf{J} \times \mathbf{B})_{x}$ as they drift dawn-to-dusk across the reconnection region. If $R_{y}$ is too small, not enough earthward acceleration is given to the ions within the time they complete the traverse $\left(\sim R_{y} / V_{i y}\right.$, $V_{i y}$ : duskward flow velocity of ions). It is known in MHD as well that the jet acceleration is reduced for a highly threedimensional system with a small $R_{y} / D$ (Ugai, 1991). On the other hand, with the scaling $(\mathbf{J} \times \mathbf{B})_{x} \propto 1 / D$ and $V_{i y} \propto 1 / D$, one concludes from a Hall-MHD point of view that it is the value of $R_{y}$ in the ion inertia unit itself that controls the degree of acceleration. Simulation results (not shown) show that it is in reality controlled by both parameters. This is another Hall effect on magnetic reconnection.

A recent study by Ergun et al. (2001) on radio wave emissions suggests that the electron-maser process firmly evidenced in the Earth's auroral region is widespread in astrophysical plasmas. Since field-aligned acceleration of electrons is requested for the electron-maser to operate, it is further suggested that electron acceleration parallel to a magnetic field is a widespread process. We have shown that a part of the intense auroral FACs in the Earth's magnetosphere are likely to be driven by the Hall term. We have also shown by a parameter survey that the role of the Hall term to produce intense FAC is rather general. FACs generated in large scale dynamics, when they are intense enough, would require fieldaligned acceleration of electrons so that the required amount of current density can be attained. As such, generation of intense FACs is a channel through which large scale dynamic phenomena can be linked to electron acceleration and energization. Our results suggest that the Hall term in magnetic reconnection may be playing a significant role universally in linking this flow of energy. While not only radio wave emissions but also other sources such as X ray emissions indicate universality of electron acceleration associated with large scale dynamic phenomena, the linkage still waits to be clarified. We may eventually find currents and structures set up by the Hall term to be playing some crucial roles in this context. A recent study by Shay et al. (1999) shows that the reconnection rate is insensitive to the initial thickness of the current sheet but is determined by the ion inertia, indicating the significance of the ion inertia effect in this aspect of the physics of reconnection as well. In this as well as in the present study, the essential fact is that appropriate ion scales are self-generated in the course of reconnection dynamics.
Acknowledgments. N. Y. and Y. Y. thank T. Nagai and M. S. Nakamura for fruitful discussion and encouragements. M. F. acknowledges stimulating discussion at Space Sciences Laboratory, UC Berkeley. M. F. is a member of the ACT-JST project 12D-1. A part of the simulations has been performed on VPP 5000 at Nagoya University Computer Center via Collaborative Research Program of STE Laboratory.

\section{References}

Baker, D. N., et al., Neutral line model for substorms: Past results and present view, J. Geophys. Res., 101, 12,975-12,986, 1996.

Birn, J. and M. Hesse, Details of current disruption and diversion in simulations of magnetotail dynamics, J. Geophys. Res., 101, 15,345-15,354, 1996.

Birn, J., et al., MHD simulations of magnetotail dynamics, J. Geophys. Res., 101, 12,939-12,949, 1996.

Daughton, W., Kinetic theory of the drift kink instability in a current sheet, J. Geophys. Res., 103, 29,429-29,437, 1998.

Ergun, R. E., et al., Electron-cyclotron maser driven by charged-particle acceleration from magnetic-field-aligned electric fields, Ap. J., 2001 (in press).

Fujimoto, M., et al., Observations of earthward streaming electrons at the trailing boundary of a plasmoid, Geophys. Res. Lett., 24, 2893-2897, 1997.

Fujimoto, M., et al., Tailward electrons at the lobe-plasma sheet interface in the near-Earth tail crossed upon dipolarizations, J. Geophys. Res., 2001 (in press).

Hesse, M. and D. Winske, Hybrid simulations of collisionless reconnection in current sheets, J. Geophys. Res., 99, 11,177-11,185, 1994.

Lottermoser, R. F., et al., Ion kinetic effects in magnetic reconnection: Hybrid simulations, J. Geophys. Res., 103, 4547-4557, 1998.

Mandt, M. E., et al., Transition to whistler mediated magnetic reconnection, Geophys. Res. Lett., 21, 73-77, 1994.

McPherron, R. L., et al., Satellite studies of magnetospheric substorms on August 15, 1968, 9, Phenomenological model for substorms, J. Geophys. Res., 78, 3131-3146, 1973.

Mitchell, D. G., et al., Current carriers in the near-Earth cross-tail current sheet during substorm growth phase, Geophys. Res. Lett., 17, 583-587, 1990

Nagai, T., et al., Structure and dynamics of magnetic reconnection during substorms with GEOTAIL observations, J. Geophys. Res., 103, 44194431, 1998 .

Nakamura, M. S. and M. Fujimoto, A three-dimensional hybrid simulation of magnetic reconnection, Geophys. Res. Lett., 25, 2917-2921, 1998.

Nakamura, M. S., M. Fujimoto, and K. Maezawa, Ion dynamics and resultant velocity space distributions in the course of magnetic reconnection, $J$. Geophys. Res., 103, 4531-4540, 1998.

Pritchett, P. L. and F. V. Coroniti, The role of the drift kink mode in destabilizing thin current sheets, J. Geomag. Geoelectr., 48, 833-848, 1996.

Scholer, M. and A. Otto, Magnetotail reconnection: Current diversion and field-aligned currents, Geophys. Res. Lett., 18, 733-736, 1991.

Shay, M. A., et al., The scaling of collisionless magnetic reconnection for large systems, Geophys. Res. Lett., 26, 2163-2166, 1999.

Sonnerup, B. U. O., Magnetic field reconnection, in Solar System Plasma Physics, Vol. 3, edited by L. T. Lazerotti, et al., Oxford, 1979.

Terasawa, T., Hall current effect on tearing mode instability, Geophys. Res. Lett., 10, 475-479, 1983.

Ugai, M., Computer modeling of three dimensional dynamics of fast reconnection, J. Plasma Phys., 45, 251-262, 1991.

Ugai, M. and W. B. Wang, Computer simulations on three-dimensional plasmoid dynamics by the spontaneous fast reconnection model, J. Geophys Res., 103, 4573-4581, 1998.

Yamade, Y., M. Fujimoto, N. Yokokawa, and M. S. Nakamura, Fieldaligned-currents generated in magnetotail reconnection: 3D Hall MHD simulations, Geophys. Res. Lett., 27, 1091-1094, 2000.

Yoon, P. H., et al., Theory and simulation of Kelvin-Helmholtz instability in the geomagnetic tail, J. Geophys. Res., 101, 27,327-27,337, 1996.

N. Yokokawa, M. Fujimoto (e-mail: fujimoto@geo.titech.ac.jp), Y. Yamade, and T. Mukai 